\title{
EVALUACIÓN EXPERIMENTAL DE LA DEGRADACIÓN FOTOCATALÍTICA DEL COLORANTE CIBACRON NAVY H-2G EMPLEANDO NANOPARTÍCULAS INDUSTRIALES DE TiO,
}

\author{
Elizabeth Deza Martía ${ }^{*}$, Ana Osorio Anaya ${ }^{a}$, Joaquín J. Manrique Fajardo ${ }^{a}$
}

\begin{abstract}
RESUMEN
En el presente trabajo se evaluó experimentalmente la degradación del colorante azul, Cibacron Navy-H2G a través de la acción fotocatalítica de nanopartículas industriales de $\mathrm{TiO}_{2}$ de la empresa española Avanzare. Las nanopartículas corresponden a la fase anatasa caracterizada por DR-X, tienen un tamaño aproximado de $26,6 \mathrm{~nm}$, medidos con TEM y $20,4 \mathrm{~nm}$, medidos con DLS. La degradación fotocatalítica se evaluó en función a la masa del fotocatalizador desde $0,1 \mathrm{~g}$ hasta $1,0 \mathrm{~g}$; a la concentración del colorante desde $20 \mathrm{ppm}$ hasta 100 ppm; y a pH desde 2 hasta 10. El tiempo de degradación fotocatalítica del colorante se monitoreo con espectrofotometría UV-Visible. Los mejores resultados del proceso se obtuvieron con una masa de $0,6 \mathrm{~g}$ de nanopartículas de $\mathrm{TiO}_{2}$, concentración de colorante de 20 ppm y a un $\mathrm{pH}$ de 4 .
\end{abstract}

Palabras clave: degradación fotocatalítica, nanopartículas de $\mathrm{TiO}_{2}$, Cibacron Navy H-2G

\section{EXPERIMENTAL EVALUATION OF THE DYE PHOTOCATALYTIC DEGRADATION CIBACRON NAVY H-2G USING INDUSTRIAL NANOPARTICLES OF TIO}

\begin{abstract}
In the present work the degradation of the blue dye, Navy H-2G cibacron was evaluated experimentally through the photocatalytic action of $\mathrm{TiO}_{2}$ industrial nanoparticles of the Spanish company Avanzare. The nanoparticles correspond to the anatase phase characterized by DR-X, have an approximate size of $26.6 \mathrm{~nm}$ measured with TEM and $20.4 \mathrm{~nm}$ measured with DLS. The photocatalytic degradation was evaluated as a function of various masses of the photocatalyst from $0.1 \mathrm{~g}$ to $1.0 \mathrm{~g}$; Depending on the concentration of the dye from $20 \mathrm{ppm}$ to $100 \mathrm{ppm}$; And depending on the $\mathrm{pH}$ from 2 to 10.5. The time of photocatalytic degradation of the dye was monitored by UV-Visible spectrophotometry. The optimal results
\end{abstract}

\footnotetext{
${ }^{a}$ Laboratorio de Nanotecnología e Innovación Tecnológica, Facultad de Química e Ingeniería Química, Universidad Nacional Mayor de San Marcos. Calle Germán Amezaga 375, Lima, Perú, lizdeza@yahoo.com.
} 
of the process were observed with a mass of $0.6 \mathrm{~g}$ of nanoparticles of $\mathrm{TiO}_{2}$, concentration of dye of $20 \mathrm{ppm}$ and at a $\mathrm{pH}$ of 4.

Key words: photocatalytic degradation, $\mathrm{TiO}_{2}$ nanoparticles, Cibacron Navy $\mathrm{H}-2 \mathrm{G}$

\section{INTRODUCCIÓN}

El colorante azul, Cibacron Navy H-2G, forma parte de la gama de colorantes reactivos utilizados en la industria textil. Se calcula que aproximadamente, entre el $1 \%$ a $15 \%$ del colorante se pierde durante los procesos de teñido y estos se liberan en las aguas residuales ${ }^{1-3}$, generando contaminación ambiental debido a los grupos azo, al dibromopropionil, monoclorotriazina, entre otros, tal como se muestra en la figura 1.

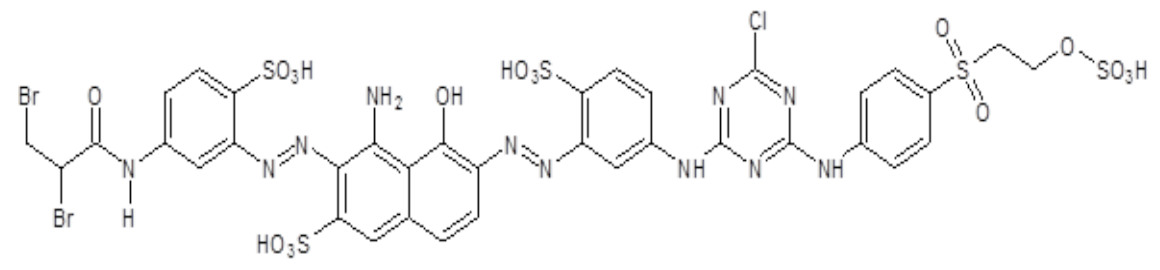

Figura 1. Estructura química del colorante Cibacron Navy H-2G.

Existen diversos métodos de descontaminación de aguas, para aplicar algún método de tratamiento se debe tener en cuenta, principalmente, la naturaleza y las propiedades fisicoquímicas de las aguas o efluentes a tratar ${ }^{4}$. Las aguas contaminadas, generalmente, pueden ser tratadas eficientemente mediante métodos biológicos, adsorción con carbón activado $\mathrm{u}$ otros adsorbentes, tratamientos químicos convencionales (oxidación térmica, cloración, ozonización, uso de oxidantes fuertes como el permanganato de potasio, entre otros.). En algunos casos, estos procedimientos resultan inadecuados, pues no logran alcanzar el grado de descontaminación final requerido por ley, es por eso que se recurre al uso de procesos o tecnologías avanzados de oxidación (PAOs, TAOs). Los PAOs se basan en procesos fisicoquímicos que pueden degradar la estructura química de los contaminantes orgánicos, generando principalmente el radical hidroxilo $(\mathrm{HO} \bullet)$ el cual es muy efectivo para oxidar compuestos orgánicos. Este radical puede ser generado por medios fotoquímicos (incluida la luz solar) o por otras formas de energía. Algunos PAOs, como la fotocatálisis heterogénea, la radiólisis y otras técnicas avanzadas, pueden transformar a contaminantes poco susceptibles a la oxidación, como iones metálicos y compuestos halogenados.

Los contaminantes orgánicos (representados por $\mathrm{RH}, \mathrm{RX}$ y $\mathrm{PhX}$ ) pueden ser oxidados por el radical hidroxilo, de acuerdo con tres mecanismos básicos: abstracción de hidrógeno, transferencia electrónica y adición electrofílica, conforme a las ecuaciones (1-3), respectivamente. 


$$
\begin{array}{lll}
\cdot \mathrm{OH}+\mathrm{RH} & \rightarrow & \mathrm{R} \cdot+\mathrm{H}_{2} \mathrm{O} \\
\cdot \mathrm{OH}+\mathrm{RX} & \rightarrow & \mathrm{RX} \cdot+\mathrm{OH}- \\
\cdot \mathrm{OH}+\mathrm{PhX} & \rightarrow & \mathrm{OH}-\mathrm{PhX} \cdot
\end{array}
$$

El radical hidroxilo $\mathrm{HO}$ - posee propiedades que le permiten atacar a los compuestos orgánicos y reaccionar $10^{6}-10^{12}$ veces más rápido que algunos oxidantes alternativos como el $\mathrm{O}_{3}$. Después del flúor, el $\mathrm{HO} \cdot$ es el oxidante más enérgico, presenta mayor velocidad de degradación con respecto al ozono $\left(\mathrm{O}_{3}\right)^{5}$, tal como se muestra en la siguiente tabla.

Tabla 1. Comparación de la velocidad de reacción entre el radical $\mathrm{HO} \bullet$ y el $\mathrm{O}_{3}$

\begin{tabular}{lcc}
\hline Compuesto orgánico & \multicolumn{2}{c}{ Velocidad de reacción $\left(\mathbf{L} \cdot \mathbf{m o l}^{-\mathbf{1}} \cdot \mathbf{s}^{-1}\right)$} \\
\cline { 2 - 3 } & HO• & $\mathbf{O}_{\mathbf{3}}$ \\
\hline Alquenos clorados & $10^{9}-10^{11}$ & $10^{-1}-10^{3}$ \\
Fenoles & $10^{9}-10^{10}$ & $10^{3}$ \\
Aromáticos & $10^{8}-10^{10}$ & $1-10^{2}$ \\
Cetonas & $10^{9}-10^{10}$ & 1 \\
Alcoholes & $10^{8}-10^{9}$ & $10^{-2}-1$ \\
Alcanos & $10^{6}-10^{9}$ & $10^{-2}$ \\
\hline
\end{tabular}

Fuente: García Ortiz, J. (2013)

La fotocatálisis es un proceso que se basa en la absorción directa o indirecta de energía radiante (visible o UV) por un sólido (el fotocatalizador heterogéneo, que normalmente es un semiconductor de banda prohibida ancha).

Los fotocatalizadores más investigados hasta el momento son los óxidos metálicos semiconductores de banda ancha $\mathrm{y}$, particularmente, el dióxido de titanio $\left(\mathrm{TiO}_{\mathrm{t}}\right)$, el cual presenta una elevada estabilidad química que lo hace apto para trabajar en un amplio rango de $\mathrm{pH}$, al mismo tiempo que es capaz de producir transiciones electrónicas por absorción de la luz en el ultravioleta cercano (UV-A).

El proceso de fotocatálisis heterogénea degrada exitosamente contaminantes presentes en diversos tipos de efluentes ${ }^{6-11}$ y constituye uno de los métodos existentes, los que se reportan en la tabla 2. 
Tabla 2. Procesos de oxidación avanzados (PAOs)

\begin{tabular}{ll}
\hline Procesos no fotoquímicos & Procesos fotoquímicos \\
\hline Ozonización alcalina $\left(\mathrm{O}_{3} / \mathrm{OH}^{-}\right)$ & Fotólisis UV de vacío \\
Ozonización con $\mathrm{H}_{2} \mathrm{O}_{2}\left(\mathrm{O}_{3} / \mathrm{H}_{2} \mathrm{O}_{2}\right)$ & $\mathrm{UV} / \mathrm{H}_{2} \mathrm{O}_{2}$ \\
Procesos fenton $\left(\mathrm{Fe}^{+2} / \mathrm{H}_{2} \mathrm{O}_{2}\right)$ & $\mathrm{UV} / \mathrm{O}_{3}$ \\
Oxidación electroquímica & Foto-fenton \\
Radiólisis $\gamma$ & $\mathrm{UV} /$ peryodato \\
Plasma no térmico & Fotocatálisis heterogénea \\
Ultrasonido & \\
Ferrioxalato y otros complejos $\mathrm{Fe}(\mathrm{III})$ & \\
\hline Fuente: Doménech X., Jardim W., Litter M. (2001)
\end{tabular}

En el presente trabajo, se evalúa la eficiencia fotocatalítica de nanopartículas de $\mathrm{TiO} 2$ de estructura anatasa del sistema tetragonal registrada en la figura 2 , se considera que su estudio es un tema emergente de las nuevas tecnologías ${ }^{12-14}$.

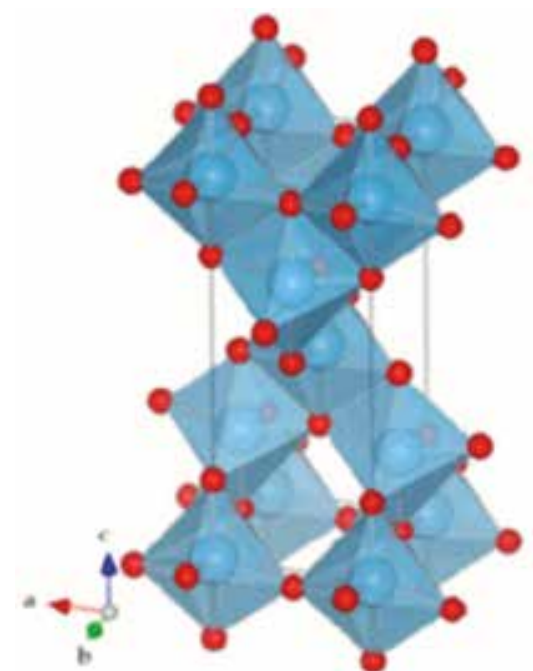

Figura 2. Estructura cristalina de la Anatasa, Sistema tetragonal $(\mathrm{a}=\mathrm{b}=3,78 \AA ; \mathrm{c}=9,51 \AA)$

\section{PARTE EXPERIMENTAL}

\section{Caracterización de la fase cristalina de las $\mathrm{NPs}^{-\mathrm{TiO}_{2}}$ por difracción de rayos $\mathrm{X}$}

Las fases cristalinas de las nanopartículas de $\mathrm{TiO}_{2}$ fueron identificadas por difracción de rayos-X (DR-X) empleando el difractómetro XPERT-PRO modelo X'Pert MPD, de la marca PANalytical, usando una fuente de $\mathrm{CuK} \alpha$ medio de $\lambda=1.54069 \AA$ Á. El rango de la medida fue realizado en 5 a $80^{\circ}$ (2Ө) con un paso de 0,03 grados y un tiempo de 50 segundos por paso, con un tiempo aproximado de 16 minutos a temperatura ambiente. La caracterización 
fue realizada en el Centro de Apoyo a la Investigación (CAI) de Difracción de Rayos X de la Universidad Complutense de Madrid.

\section{Medición del tamaño de nanopartículas de $\mathrm{TiO}_{2}$ por TEM y DLS}

La distribución del tamaño de partícula se caracterizó por microscopía electrónica de transmisión (TEM) con el equipo JEOL, modelo JEM-2100HT. Todas las mediciones TEM se realizaron con una suspensión de $100 \mathrm{mg} / \mathrm{L}$ para cada medición, $1 \mathrm{~mL}$ de la solución coloidal se colocó sobre una rejilla de cobre recubierta de carbono y se dejaron secar durante 5 minutos. La medición se realizó en el Centro Nacional de Microscopía Electrónica de la Universidad Complutense de Madrid.

Respecto al método de la dinámica de dispersión de luz (DLS), es una técnica fisicoquímica empleada para la determinación de la distribución de tamaños de partículas en suspensión, el equipo utilizado es el Nicomp N3000, la medición se realizó en el Laboratorio de Nanotecnología e Innovación Tecnológica de la Facultad de Química e Ingeniería Química de la Universidad Nacional Mayor de San Marcos.

\section{Metodología experimental de la degradación del colorante por fotocatálisis}

$\mathrm{Se}$ emplearon diferentes pesos de nanopartículas de $\mathrm{TiO}_{2}\left(\mathrm{NPs}-\mathrm{TiO}_{2}\right)$ en polvo, según referencia de la tabla 3.

Tabla 3. Masas de fotocatalizador empleadas en la parte experimental

\begin{tabular}{|c|c|c|c|c|c|c|c|}
\hline $\mathrm{NPs} \mathrm{TiO}_{2}$ & M1 & M2 & M3 & M4 & M5 & M6 & M7 \\
\hline Masa (g) & 0,1 & 0,2 & 0,3 & 0,4 & 0,5 & 0,6 & 1,0 \\
\hline
\end{tabular}

Las NPs- $\mathrm{TiO}_{2}$ o fotocatalizador se colocó dentro de un reactor conteniendo $1 \mathrm{~L}$ de solución 20ppm del colorante azul Cibacron Navy-H2G e irradiados con luz UV de una lámpara de mercurio de $250 \mathrm{~W}$ marca Tungsram, con burbujeo de oxígeno con agitación constante y a temperatura ambiente hasta la observación de la decoloración, el sistema de trabajo se aprecia en la figura 3.

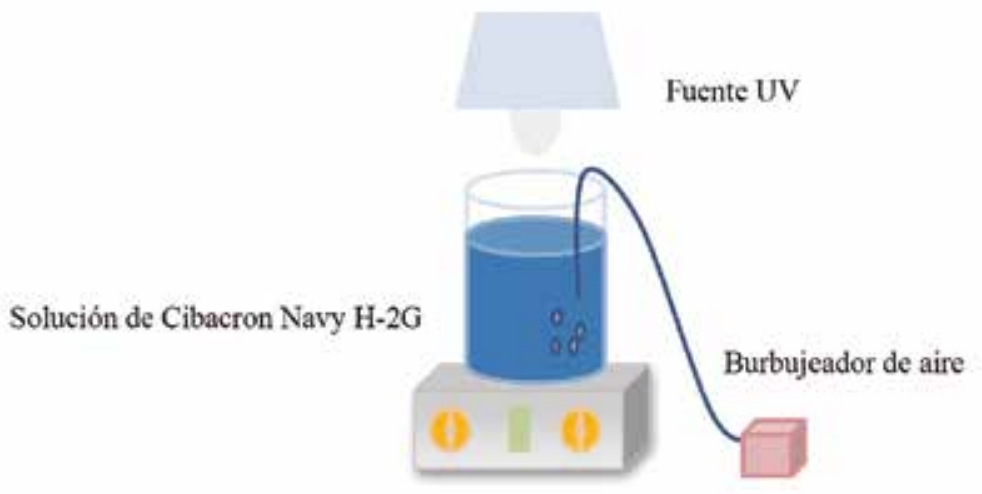

Figura 3. Reactor empleado en el desarrollo experimental de la fotocatálisis 
Una vez evaluada la eficiencia del sistema en función al peso de las $\mathrm{NPs}_{-}-\mathrm{TiO}_{2}$, se tomó como variable la concentración del colorante según se especifica en la tabla 4.

Tabla 4. Concentraciones del colorante azul empleadas en el desarrollo experimental

\begin{tabular}{cccccc}
\hline Colorante & $\mathrm{C} 1$ & $\mathrm{C} 2$ & $\mathrm{C} 3$ & $\mathrm{C} 4$ & $\mathrm{C} 5$ \\
\hline $\begin{array}{c}\text { Concentración } \\
(\mathrm{ppm})\end{array}$ & 20 & 40 & 60 & 80 & 100 \\
\hline
\end{tabular}

Luego de evaluar los resultados en función a la concentración de la solución del colorante azul Cibacron Navy H-2G, se evaluó la acción fotocatalítica en función al pH de valores: 2, $4,6,8$ y 10 .

\section{Monitoreo de la degradación fotocatalítica con mediciones en el rango de UV-Visible.}

Las mediciones de la degradación fotocatalítica del colorante se realizó en el equipo espectrofotómetro PG INSTRUMENTS modelo T80+, las mediciones se realizaron cada 10 minutos hasta la observación de la decoloración completa del colorante azul.

\section{RESULTADOS Y DISCUSIÓN}

En la figura 4 se observa el difractograma de las nanopartículas de $\mathrm{TiO}_{2}$ Avanzare, se muestran claramente las líneas de difracción en los ángulos $2 \theta=25^{\circ}, 48^{\circ}, 54^{\circ}, 55^{\circ}, 62^{\circ}, 71^{\circ}$ y $75^{\circ}$, característicos de la fase anatasa (tetragonal) del óxido de titanio, y también los ángulos pertenecientes a la fase rutilo en los ángulos $2 \theta=27^{\circ}, 36^{\circ}, 41^{\circ}, 44^{\circ}$ y $57^{\circ}$ que se encuentran en menor porcentaje. Se comprueba que las dos fases están presentes, en un porcentaje de $85 \%$ y $15 \%$ registrado en la tabla 5 .

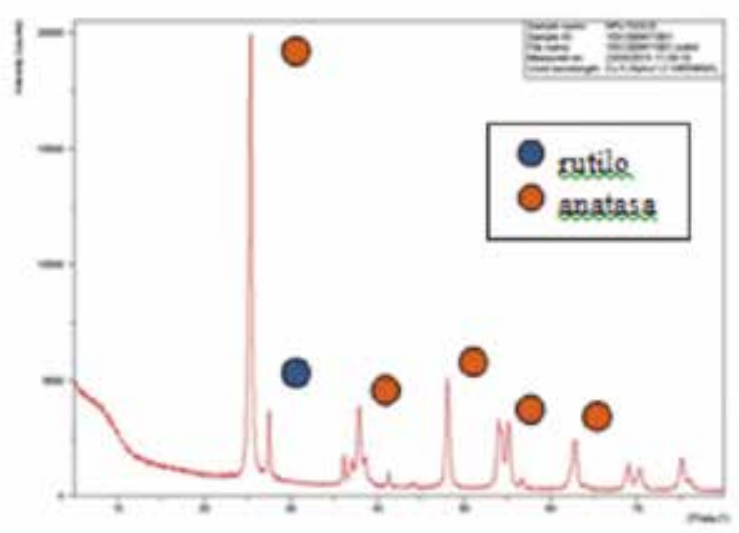

Figura 4. Difractograma de las nanopartículas de $\mathrm{TiO}_{2}$ Avanzare 
La anatasa como el rutilo presentan el mismo sistema cristalino, la diferencia entre la anatasa y el rutilo radica en el parámetro c de sus celdas, siendo el de la anatasa tres veces mayor que la del rutilo.

Tabla 5. Composición porcentual de las NPs- TiO2 medido con el difractómetro XPERT-PRO

\begin{tabular}{cccc}
\hline Código de referencia & Nombre de mineral & Fórmula química & $\begin{array}{c}\text { Semicuantificación } \\
(\mathbf{\%})\end{array}$ \\
\hline PDF 00-021-1272 & Anatasa & $\mathrm{TiO}_{2}$ & 85 \\
PDF 00-021-1276 & Rutilo & $\mathrm{TiO}_{2}$ & 15 \\
\hline
\end{tabular}

Con respecto a la medición del tamaño de las nanopartículas de $\mathrm{TiO}_{2}$ se realizó directamente de las microfotografías del TEM, midiendo las partículas con la escala de la resolución y se analizó alrededor de 80 nanopartículas para su evaluación estadística.
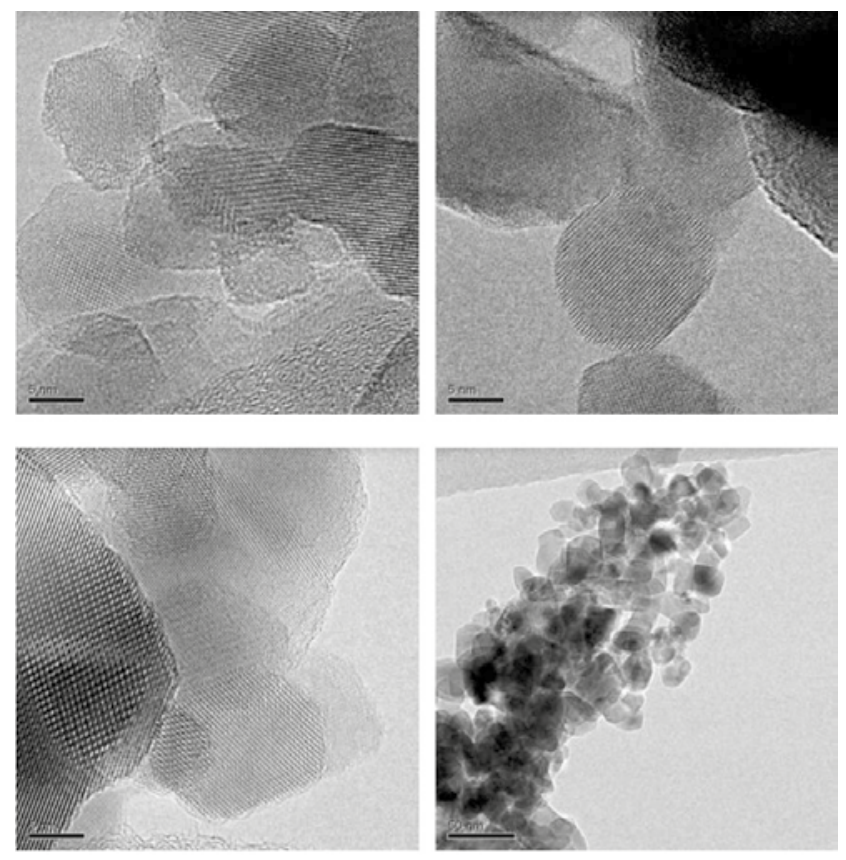

Figura 5. Medición del tamaño por distribución de $\mathrm{NPs}_{\text {de }} \mathrm{TiO}_{2}$ de la empresa Avanzare. 


\begin{tabular}{cc}
\hline $\begin{array}{c}\text { TEM (microscopio } \\
\text { electrónico de transmisión) }\end{array}$ & Tamaño de partícula (nm) \\
\hline $\mathrm{TiO}_{2}$ Avanzare & $26,64 \pm 6,23$
\end{tabular}

Por otro lado, las mediciones realizadas por DLS indican que las nanopartículas de $\mathrm{TiO}_{2}$ presentan en cuanto a la intensidad de tamaño de partículas dos distribuciones en promedio de $21,2 \mathrm{~nm}$ y $250,5 \mathrm{~nm}$, tal como se aprecia en la figura 6 (a). En cuanto a la distribución del volumen se presenta igualmente dos distribuciones a $20,4 \mathrm{~nm}$ y la otra de $239 \mathrm{~nm}$ registradas en la figura 6 (b). Respecto a la distribución del número de partículas se observa monodispersidad en $20,1 \mathrm{~nm}$ correspondiente al tamaño hidrodinámico de las nanopartículas de $\mathrm{TiO} 2$ apreciadas en la figura 6 (c).

De acuerdo a las mediciones realizadas, se puede concluir que existe un mínimo de partículas correspondientes al tamaño entre $250,5 \mathrm{~nm}$ y $239 \mathrm{~nm}$ que representan un porcentaje de $0,5 \%$ en tanto que las nanopartículas de tamaños entre $20,4 \mathrm{~nm}$ y $20,1 \mathrm{~nm}$ representan el $99,5 \%$.

Con los resultados obtenidos se puede apreciar que las partículas son monodispersas en un alto porcentaje.
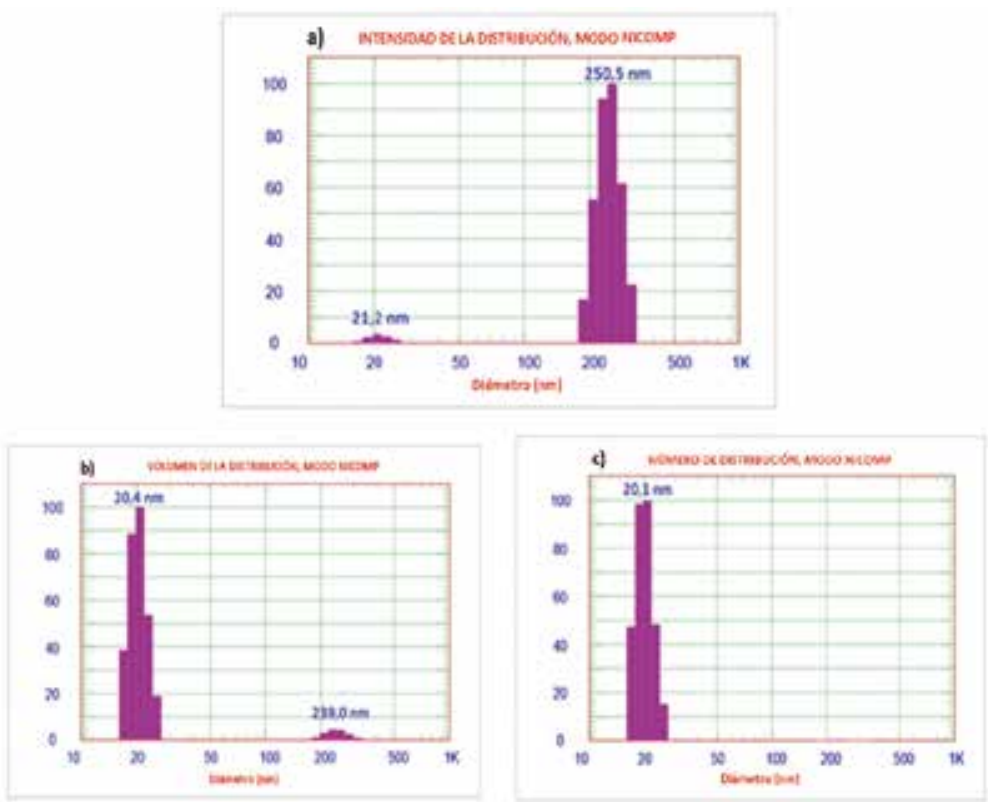

Figura 6. Determinación de la dispersión de nanopartículas: (a) intensidad, (b) volumen y (c) número de nanopartículas de $\mathrm{TiO}_{2}$ Avanzare. 
La fotodegradación, empleando sólo radiación UV, es casi nula en ausencia del catalizador, como se puede apreciar en la figura 7.

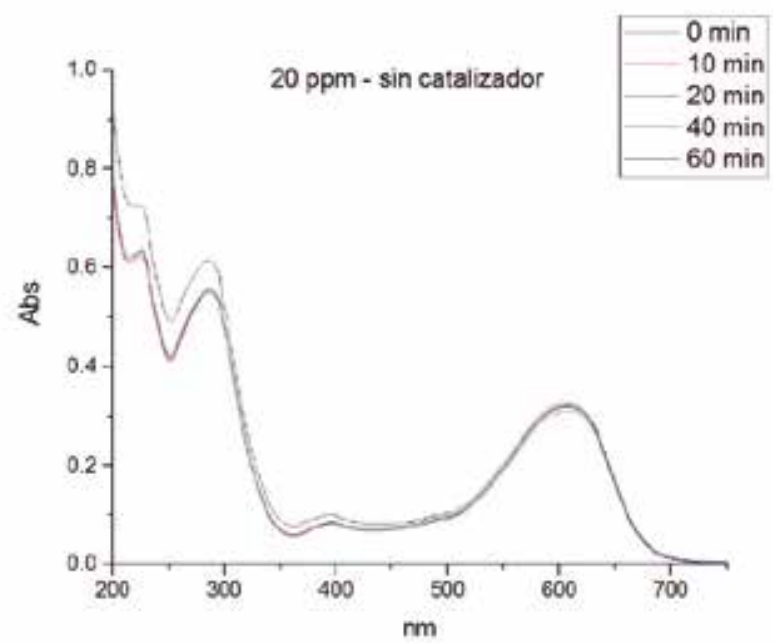

Figura 7. Espectro de absorción UV-Visible del colorante Cibacron Navy H-2G de concentración $20 \mathrm{ppm}$ sin empleo de fotocatalizador.

Al evaluar la influencia del peso del fotocatalizador se obtiene mayor velocidad de degradación al utilizar 0,6 g de TiO2 con una decoloración a los 10 minutos y a la desaparición de las bandas presentes en el rango ultravioleta a los 30 minutos como se aprecia en la figura 8 .

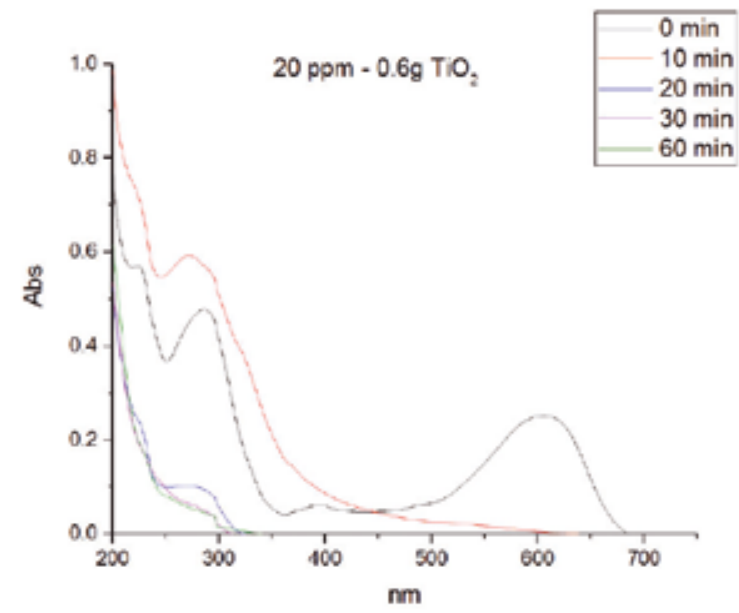

Figura 8. Espectro de absorción UV-Visible del colorante Cibacron Navy H-2G de concentración $20 \mathrm{ppm}$ en presencia de $0,6 \mathrm{~g}$ de fotocatalizador. 
En la figura 9 se observa que a medida que se aumenta la concentración de colorante el tiempo de decoloración aumenta a los 130 minutos y las bandas que se encuentran en la región ultravioleta se degradan lentamente por lo que se requiere más de 3 horas para degradarse completamente.

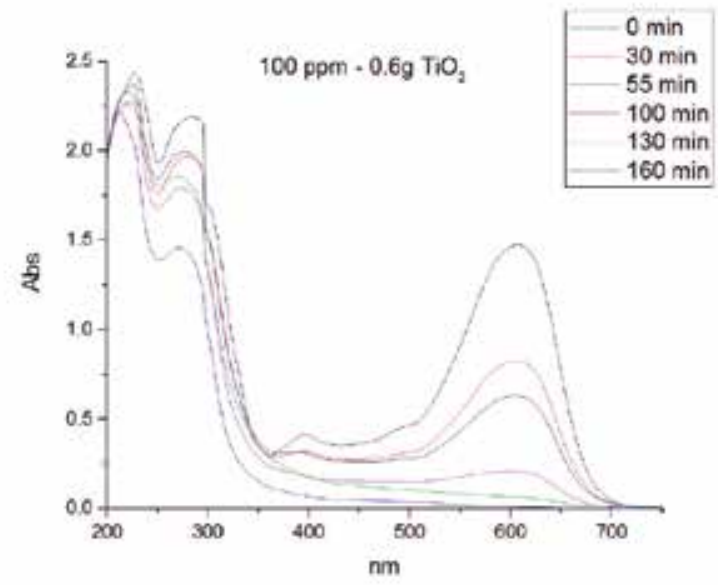

Figura 9. Espectro de absorción UV-Visible de 100 ppm de colorante Cibacron Navy $\mathrm{H}-2 \mathrm{G}$ con $0,6 \mathrm{~g}$ nanopartículas de $\mathrm{TiO}_{2}$ Avanzare.

La última evaluación experimental fue en función al $\mathrm{pH}$, comprobándose que a medida que disminuye el $\mathrm{pH}$, la degradación fotocatalítica aumenta, lo contrario sucede a $\mathrm{pH}$ básico, se determinó que la velocidad de degradación aumenta a pH 4, se observó que a los 20 minutos la solución estaba decolorada y los componentes cuyas bandas de absorción están en el rango ultravioleta se degradaron en 40 minutos, tal como se observa en la figura 10.

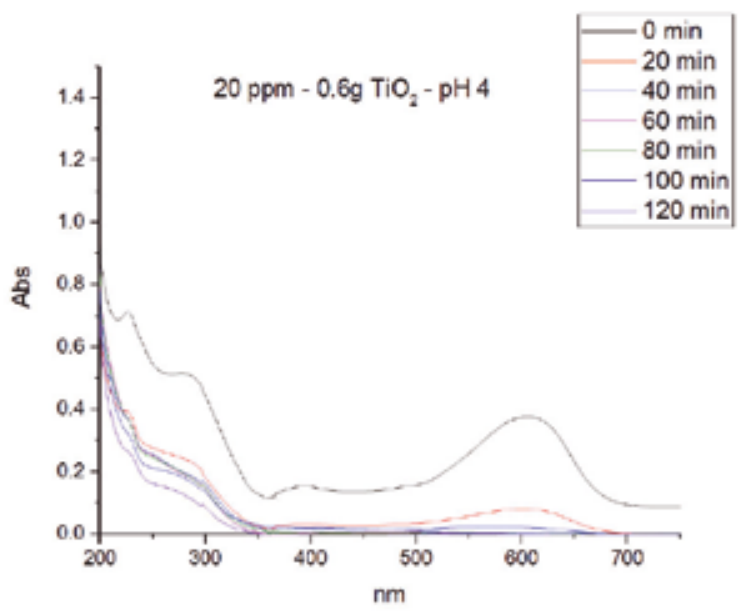

Figura 10. Espectro de absorción UV-Visible de 20 ppm de colorante Cibacron Navy $\mathrm{H}-2 \mathrm{G}$ con $0,6 \mathrm{~g}$ nanopartículas de $\mathrm{TiO} 2$ Avanzare a $\mathrm{pH} 4$. 
En la figura 11 se observa que a $\mathrm{pH}$ básico la velocidad de degradación disminuye observándose la decoloración a los 100 minutos y a los 120 minutos la desaparición de las bandas del rango del ultravioleta.

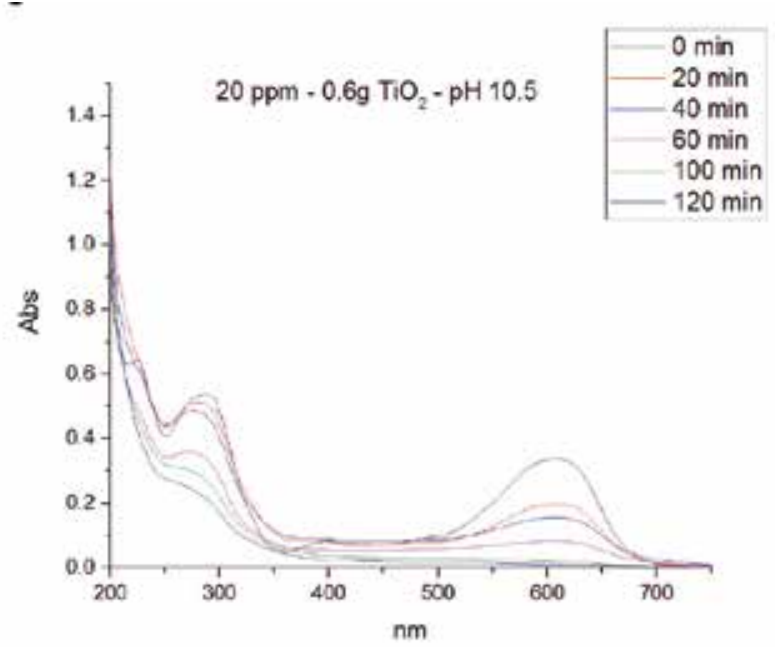

Figura 11. Espectro de absorción UV-Visible de 20 ppm de colorante Cibacron Navy $\mathrm{H}-2 \mathrm{G}$ con $0,6 \mathrm{~g}$ nanopartículas de $\mathrm{TiO}_{2}$ Avanzare a pH 10,5.

En las figuras 8, 9, 10 y 11 se observa que es mayor la velocidad de decoloración, debido principalmente a la ruptura de los enlaces azo, pero los grupos aromáticos que absorben en la región del UV, toman mayor tiempo en degradarse.

Las bandas que se encuentran a 208 y $286 \mathrm{~nm}$ corresponden a la presencia de anillos bencénicos en la molécula del colorante Cibacron Navy H-2G. Estas bandas desaparecen más rápidamente a $\mathrm{pH}$ ácido como se observa en la figura 10.

Como se sabe, al inicio de la reacción de fotocatálisis ocurre la adsorción del colorante en la superficie del $\mathrm{TiO}_{2}$. La cantidad de colorante adsorbido es mayor en medio ácido, esto se puede atribuir a la influencia del $\mathrm{pH}$ de la solución tanto en la superficie del $\mathrm{TiO}_{2}$ como en el grado de ionización de la molécula orgánica del colorante. En general se puede correlacionar la disminución del $\mathrm{pH}$ con el aumento de la cantidad de $\mathrm{TiOH}_{2}^{+}$el cual es responsable de la adsorción del colorante en la superficie del fotocatalizador ${ }^{15}$. Uno de los mecanismos propuestos, que describen cómo se degradan los compuestos orgánicos, indica que los huecos fotogenerados $\left(\mathrm{h}^{+}{ }_{\mathrm{bv}}\right)$ oxidan directamente a los compuestos adsorbidos en la superficie del semiconductor ${ }^{16}$. A pH ácido se favorece la formación de huecos ${ }^{17}$, y estos huecos en la superficie del $\mathrm{TiO}_{2}$ provocan la fijación de la molécula del colorante, ello explica que la degradación en el rango UV ocurra en menor tiempo a $\mathrm{pH}$ ácido. Los anillos aromáticos que absorben en el UV se fijan a la superficie mediante un ataque electrofílico como se observa en las figuras 12 y 13. 


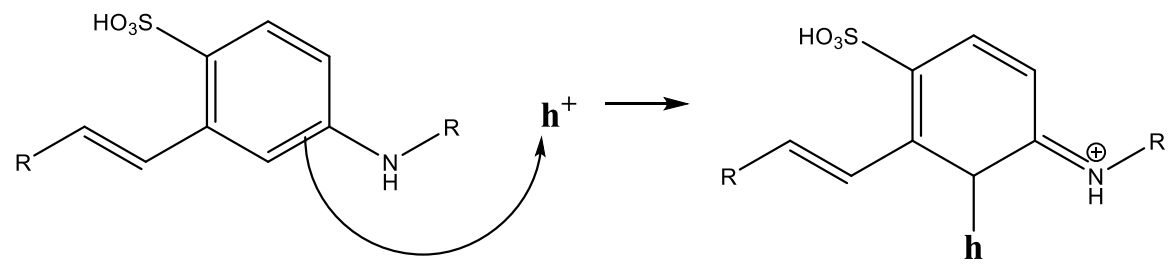

Figura 12. Fijación del anillo aromático sobre la superficie del $\mathrm{TiO}_{2}$. Ataque electrofílico sobre los huecos fotogenerados $\left(\mathrm{h}^{+}{ }_{\mathrm{bv}}\right)$

La etapa subsiguiente podría ser la ruptura de la estructura cíclica resultante (no aromática) debido a la acción de los radicales $\bullet \mathrm{OH}$ sobre el enlace señalado, perdiéndose irreversiblemente los anillos aromáticos que dan origen las bandas del UV.

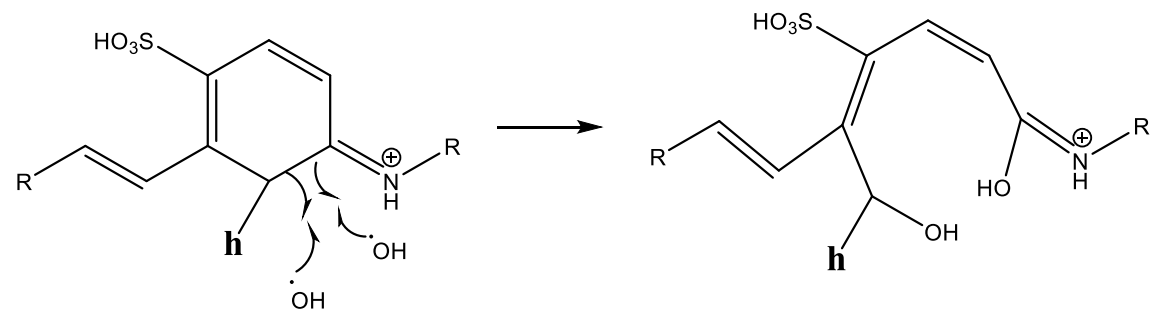

Figura 13. Ruptura del anillo aromático por acción de radicales $\bullet \mathrm{OH}$

Por otro lado, los radicales $\bullet \mathrm{OH}$ se obtienen debido a que los huecos oxidan el agua adsorbida según la siguiente ecuación:

$$
\mathrm{H}_{2} \mathrm{O}+\mathrm{h}^{+} \cdot \mathrm{OH}+\mathrm{H}^{+}
$$

En general, se supone que en solución alcalina es más fácil generar radicales $\bullet \mathrm{OH}$ oxidando los iones hidróxido de mayor disponibilidad en la superficie del $\mathrm{TiO}_{2}$, mejorando la eficiencia del proceso, pero se debe notar que en solución alcalina hay una repulsión coulómbica entre la superficie negativa del fotocatalizador y los aniones hidróxido. Este hecho puede retrasar la formación del $\bullet \mathrm{OH}$ y así disminuir la actividad fotocatalítica; como se ve en los resultados de la figura 11. Se presume que los productos finales de la degradación, además de $\mathrm{CO}_{2}, \mathrm{H}_{2} \mathrm{O}$, podría contener aniones ${ }^{18}$ como $\mathrm{NO}_{3}^{-}, \mathrm{SO}_{4}{ }^{2-}$, entre otros, menos dañinos al ambiente que los grupos orgánicos del colorante. 


\section{CONCLUSIONES}

A través de la evaluación experimental de la degradación fotocatatalítica del colorante textil azul, Cibacron Navy H-2G empleando como fotocatalizadores nanopartículas de $\mathrm{TiO}_{2}$ de la fase anatasa, con un tamaño promedio de $24 \mathrm{~nm}$, medidos por TEM, y con controles de UVVisible en función al tiempo de la decoloración y degradación del colorante se determina que los parámetros que aumentan la velocidad del proceso corresponde a $0,6 \mathrm{~g}$ de nanopartículas de $\mathrm{TiO}_{2}, 20$ ppm de colorante y $\mathrm{pH} 4$.

\section{AGRADECIMIENTO}

Agradecemos a INNOVATE PERÚ, CONVENIO N 134-FINCyT-IA-2013. Los ensayos fueron realizados en los laboratorios de Nanotecnología en Innovación Tecnológica (LabNanoInTec-FQIQ-UNMSM) y en el Laboratorio de Catálisis y Medio Ambiente de la Facultad de Química e Ingeniería Química de la Universidad Nacional Mayor de San Marcos.

\section{BIBLIOGRAFÍA}

1. Rani B, Kumar V, Singh J, Bisht S, Teotia P, Sharma S, Kela R. Bioremediation of dyes by fungi isolated from contaminated dye effluent sites for bio-usability. Braz $\mathbf{J}$ Microbiol. 2014; 45(3):1055-63.

2. Dutta AK, Maji SK, Adhikary B. $\gamma$-Fe2O3 nanoparticles: An easily recoverable effective photo-catalyst for the degradation of rose bengal and methylene blue dyes in the wastewater treatment plant. Mater Res Bull. 2013; 49: 28-34.

3. Predescu A, Nicolae A. Adsorption Of $\mathrm{Zn}, \mathrm{Cu}$ And $\mathrm{Cd}$ From Waste Waters By Means Of Maghemite Nanop Articles. UPB Sci Bull. Series B: Chemistry and Materials Science. 2012; 74: 255-264.

4. Peral J, Domenech X, Ollis DF. Heterogeneous Photocatalysis for Purification, Decontamination and Deodorization of Air. J Chem Technol Biotechnol. 70; 1997: 117140.

5. García Ortiz J. Depuración de aguas contaminadas con plaguicidas empleados en cultivos de caña de azúcar en Colombia. [tesis para optar el grado de Master en tratamientos de aguas]. Valencia: Universidad Politécnica de Valencia; 2013.

6. Duarte CJ, Forerp VF. Evaluación de un sistema de oxidación por fotocatálisis para la degradación del plaguicida thiodan 35 ec (i.a. Endosulfán) a nivel de laboratorio. [tesis para optar el grado de ingeniero ambiental y sanitario]. Bogotá: Universidad de la Salle; 2008.

7. Al-Rasheed, Radwan. Water treatment by heterogeneous photocatlysis, an overview. Saline Water Desalination Institute and Saline Water Conversion Corporation, Arabia Saudita; 2005.

8. Blanco J, Rodríguez SM, Estrada CA, Bandala ER, Gelover S, Leal T. Purificación de aguas por fotocatálisis heterogénea. Cap 3. Almería; 2005. 
9. Mills A, Le Hunte S. An overview of semiconductor photocatalysis. J Photochem Photobiol A. 1997; 108:1-35.

10. Degremont G. Water Treatment Handbook. 6th Ed. París: Lavoisier Publishing; 1991.

11. Ramesh BB, Parande AK, Raghu S, Prem Kumar T. Cotton Textile Processing: Waste Generation and Effluent Treatment. J. Cotton Sci. 2007, 11, 141-153.

12. Ram C, Kant R, Singh V. Photocatalytic Degradation of Textile Dye by Using Titanium Dioxide Nanocatalyst. Int J Theor \& Appl Sci. 2012, 4(2): 82-88.

13. Renge VC, Khedkar SV, Bhoyar KS. Micropollutant removal from waste water treatment plant - a review. IJAET. 2012; 3(4): 27-30.

14. Blanco Gálvez, Julián; Malato Rodríguez, Sixto. Purificación de aguas por fotocatálisis heterogénea: estado del arte. [Internet]; 2003. [citado el 11 de noviembre del 2016] Disponible en: http://www.cnea.gov.ar/sites/default/files/08cap03.

15. Barka N, Qourzal S, Assabbane A. Photocatalytic Degradation of an Azo Reactive Dye, Reactive Yellow 84, in Water Using an Industrial Titanium Dioxide Coated Media Arabian J Chem. 2010; 3: 279-283.

16. Gerischer H, Heller A. The role of oxygen in photooxidation of organic molecules on semiconductor particles. J Phys Chem. 1991; 95(13): 5261-5267.

17. Minero C, Pelizzetti E, Piccini P, Vincenti M. J Phys Chem B. 1999; 103: 6400.

18. Clavijo Penagos J. Sobre las reacciones de eliminación de compuestos contaminantes de aguas por fotocatálisis con TiO2 / UV: caso azul de metileno. Revista Elementos. 2013; 3: $149-156$. 